\title{
Clinical Manifestations of Adverse Effects of Statins, Oxidative Stress and Possible Role of Antioxidants in Prevention?
}

\author{
Jan Fedacko $^{1}$, R. B. Singh ${ }^{2, *}$, S. Chaithiraphan ${ }^{3}$, Viola Vargova ${ }^{1}$, Brian Tomlinson ${ }^{4}$, \\ Fabien De Meester ${ }^{5}$, Anna Gvozdjakova ${ }^{6}$ and Sven Moesgaard ${ }^{7}$ \\ ${ }^{I}$ Faculty of Medicine, Safaric University, Kosice, Slovakia \\ ${ }^{2}$ Halberg Hospital and Research Institute, Moradabad, India \\ ${ }^{3}$ Chaophya Hospital, Bankok, Thailand \\ ${ }^{4}$ Prince of Wales Hospital, Chinese University of Hong Kong, Hong Kong \\ ${ }^{5}$ Tsim Tsoum Institute, Krakow, Poland \\ ${ }^{6}$ Comenius University School of Medicine, Bratislava, Slovak Republic \\ ${ }^{7}$ Pharma Nord, Vejle, Denmark
}

\begin{abstract}
Background: There is evidence that statins are potent and effective agents with several pleiotropic effects for treatment of coronary artery disease(CAD). Statin may have adverse effects, if given in higher doses and in combinations.

Methods: Internet search till 2009 and discussion with colleagues.

Results: Statins are wonder drugs influencing wide range of physiological, biochemical,and biological functions. This list appears to be quite large and includes hypolipidemic, vasodilative, antithrombotic, antioxidant, antiinflammatory, antiproliferative, anticoagulant, agiogenic and bone formation inducing functions. Myopathy is the most frequent side effect of statins and in some cases may have a form of severe rhabdomyolysis. Less common adverse effects include hepatotoxicity, peripheral neuropathy, impaired myocardial contractility and autoimmune diseases. Rare manifestations of statin intolerance may be; pulmonary, psychiatric, ophthalmic and amyotropic lateral sclerosis. The risk of these unfavorable effects is largely outweighed by great reduction of cardiovascular events in statin users. The spectrum of statin-related myopathy ranges from common but clinically benign myalgia to rare but life-threatening rhabdomyolysis. Observational studies suggest that myalgia can occur in up to $10 \%$ of persons prescribed statins, whereas rhabdomyolysis continues to be rare. The mechanisms of statin-related myopathy are unclear. The criteria of diagnosis of myopathy do not bother about the symptoms of patients and oxidative stress, in absence of raised muscle enzymes.Coenzyme Q10 and other antioxidants are not considered in the prophylaxis of statin toxicity. Because one study showed no decrease in CoQ in the muscle in presence of toxicity, although several studies indicate a reduction in serum levels.

Conclusion: Statins would be used more commonly in near future due to their cholesterol lowering and antiinflammatory effects. There would be a marked increase in the Number of patients with statin toxicity. Several studies have reported a significant reduction in the serum CoQ in patients receiving statins.Such concern has also been expressed by the International College of Cardiology in their meeting in April 2002 and 2005 and 2009, proposing that coenzyme Q10 and other antioxidants should be considered in the prevention as well as treatment of statin intoxication.
\end{abstract}

Keywords: Myopathy, hepatotxicity, neuropathy, statin toxicity, ubiquinone, cerivastatin, cholesterol, inflammation.

\section{INTRODUCTION}

Although statins are generally well-tolerated, adverse effects may occur in some patients. These effects result from impaired protein prenylation, deficiency of coenzyme Q involved in mitochondrial electron transport, genetic varia-

*Address correspondence to this author at the Halberg Hospital and Research Institute, Civil Lines, Moradabad-10(UP)244001, India;

Tel/Fax: 0091591 2417437; E-mail: icn2005@ sancharnet.in tions and antioxidant protection, abnormal protein glycosylation due to dolichol shortage, or deficiency of selenoproteins [1-7]. Statins inhibit 3-hydroxy-3-methylglutarylcoenzyme A (HMG-CoA) reductase, the rate-limiting enzyme in cholesterol biosynthesis, which converts HMG-CoA to mevalonate. Statins lower plasma low-density lipoprotein (LDL) cholesterol by causing intracellular cholesterol depletion and upregulating the expression of LDL receptors [4-7]. Apart from cholesterol, mevalonate is also the substrate for the synthesis of nonsteroid isoprenoids including farnesylpy- 
rophosphate, geranylgeranylpyrophosphate (both attached to small GTP-binding proteins by protein prenyltransferases), coenzyme Q, dolichol, isopentenyladenosine, etc. Depletion of these isoprenoids results in so called "pleiotropic" effects of statins which are independent of cholesterol lowering.

The most frequent side effect of statins is myopathy and in some cases may have a form of severe rhabdomyolysis. Less common adverse effects include hepatotoxicity, peripheral neuropathy, impaired myocardial contractility and autoimmune diseases [5-7]. The risk of these unfavorable effects is largely outweighed by marked reduction of cardiovascular events in statin users. Rare manifestations of statin intolerance may be; pulmonary, psychiatric, ophthalmic and amyotropic lateral sclerosis [8-12]. However, due to increasing number of patients taking statins, monitoring for any side effects, intense research to recognize their mechanisms and to identify susceptible patients, as well as rational management of these complications are mandatory to further improve safety of these excellent drugs.

Statin-related myopathy is a clinically important cause of statin intolerance and discontinuation. The spectrum of statin-related myopathy ranges from common but clinically benign myalgia to rare but life-threatening rhabdomyolysis [13-17]. The mechanisms of statin-related myopathy are unclear. Options for managing statin myopathy include statin switching, particularly to fluvastatin or low-dose rosuvastatin; nondaily dosing regimens; nonstatin alternatives, such as ezetimibe and bile acid-binding resins; and coenzyme Q10 supplementation [18-20]. Few of these strategies have highquality evidence supporting them.

\section{EXTENT OF STATIN INTOLERANCE}

Observational studies suggest that myalgia can occur in up to $10 \%$ of persons prescribed statins, whereas rhabdomyolysis continues to be rare. A warning letter was issued by FDA on May 1,2000 about liver failure as an adverse reaction of treatment with statins,based on a report of half of the 62 patients dying due to liver failure [13-17]. It has been suggested that muscle injury can occur in $1 \%$ of users of statins which in the United States alone would equal 130,000 patients with muscle toxicity manifestations [3]. Because statin-related myopathy will probably become more common with greater numbers of persons starting high-dose statin therapy and the increasing stringency of low-density lipoprotein cholesterol level targets, research to better identify patients at risk for statin myopathy and to evaluate management strategies for statin-related myopathy is warranted [1317]. Statins are likely to be more widely used in most European countries, although data on the extent of this are not generally available [1]. Use of statins across Europe is extensive but variable. The maximum use is in Norway, with over five times, the per capita use than in Italy, which has the lowest use. Commonly used statins in the European union are simvastatin and atorvastatin. The JUPITER study would further increase the use of statins [13,14]. The subjects included in this trial were, $57 \%$ with hypertension and $41 \%$ with the metabolic syndrome which may be considered to have high risk $[13,14]$. Such patients are advised to have a target LDL-cholesterol of less than that specified in current guidelines, because the most likely 'physiological' LDL- cholesterol level for humans is probably $<60 \mathrm{mg} / \mathrm{dL}(1.54$ $\mathrm{mmol} / \mathrm{L}$ ). In JUPITER trial, $75 \%$ of the participants had baseline LDL-cholesterol levels of $>94 \mathrm{mg} / \mathrm{dL}(2.4 \mathrm{mmol} / \mathrm{L})$. The results of JUPITER indicate that all patients at some risk would receive statins in future which would be advocated by statin experts in the future guidelines.

\section{RISK FACTORS FOR STATINS TOXICITY}

Myositis can occur to patients with complex medical problems who are taking multiple medications. High dose statin monotherapy, combination with other medications such as cyclosporine, fibrates, macrolide antibiotics, certain antifungal drugs and niacin are important predisposing factors of statin toxicity [5-7, 21-38]. Higher risk patients for statin myopathy are those older than 80 , with a small body frame, on higher statin doses, on other medications, or with other systemic diseases including hepatic or renal diseases, diabetes mellitus, or hypothyroidism. One of the most important factors for predicting risk of myopathy is also way of metabolization of statins. Most of them are metabolized by cytochrome P 450 family with the exception of pravastatin [39-41]. Lovastatin, simvastatin and atorvastatin are metabolized by cytochrome P 450 P3A like nifedipine, felodipine, amiodarone, macrolide antibiotics, cisapride, omeprazole etc. Fluvastatin is metabolized by cytochrome P 450 P2C9 subfamily like diclofenac, warfarin or tolbutamide etc. That is explanation why during treatment with pravastatin there were less muscle complications observed. The next explanation in favour of pravastatin is that it is hydrophilic statin in contrast to all other above named statins which is not causing "increased fluidity of muscle cell membranes" responsible for the increased risk of myopathy development [41]. Poor coenzyme Q10 status and antioxidant status in the body are also a predisposing factors for statin toxicity [18-20, 42, 43].

Genetic predisposition to statin myopathy is a rapidly expanding area of investigation. This review summarizes the latest information on genetic risk factors associated with statin-induced myopathy [4]. Genetic determinants involved in both pharmacokinetics of statins and metabolic muscle diseases are discussed. Data are provided on the prevalence of statin use in the United States; incidence of associated myopathy; terminology relating to statin myopathy and genetic susceptibility; and common myths surrounding this disorder. Technological advances now make it possible to identify genetic variation in the human genome that reveals disease-causing mutations and single nucleotide polymorphisms associated with disease. More than 30,000 individuals in the United States suffer from severe life-threatening symptoms of statin-induced myopathy that may, in some cases, persist long after the cessation of therapy. Genes of interest include those involved in the pharmacokinetics of the statin response, muscle atrophy, exercise intolerance, pain perception, and mitochondrial energy metabolism. Genetic analysis for variants and disease-causing mutations relevant to statin myopathy will provide predisposition testing for this and other drug-induced disorders. This testing will become an integral part of personalized medicine that will contribute to the safe and informed use of selected drugs and improved compliance. 


\section{CLINICAL MANIFESTATIONS OF TOXICITY}

The actual longterm adverse manifestations of statins, especially on general use are not known. Only 7 statins have been introduced by the Food and Drug Administration of United States, for general use between 1987 and 2008. They are: lovastatin(Mevacor), pravastatin (Pravachol), simvastatin (Zocor), fluvastatin (Lescol), atorvastatin (Lipitor), cerivastatin (Baycol)and rosuvastatin (Superstatin) (Table 1) [1-3]. The Japanese statin (Pitavastatin, Livalo) and sequalestatin are likely to be introduced in near future. Table 2 shows various adverse manifestations of statins during randomized, controlled trials. Lovastatin is about to become available as a generic product. All of these drugs reduce cholesterol through inhibition of HMG-CoA reductase. However,there are differences in other characteristics such as potency, lipophilicity, pharmacokinetics and in some of the nonlipid lowering properties. These controversial issues remain unresolved [1-3]. Higher levels of trasaminases elevation with statin therapy are dose dependent and occur in 0.5 to $2.0 \%$ of cases. Reversal of increased liver enzymes is frequently observed with a decrease in dose and elevations are often not seen with either rechallenge or on administration of other statin [42]. It is also observed that statins do not have adverse effects to the outcome in persons with chronic transeminase elevations due to viral hepatitis [1]. Statin therapy of hyperlipidemia may some times improve increase in transeminase in patients with fatty liver [1]. From a safety perspective, both clinical trials and post-marketing surveillance have demonstrated that statins are generally well tolerated, with rare serious adverse drug reactions (ADRs) that affect mainly muscle, liver and kidney. Treatment with statins is also implicated in the greater incidence of some rare mamifestations; ophthalmic, neurological, pulmonary, neoplasia , peripheral neuropathies and some psychiatric disturbances without such evidence in the large blinded controlled trials [2-17, 21-33].

\section{MUSCLE DAMAGE AND MYOPATHY}

Myopathy is a general term referring to any disease of muscles and myalgia means muscle pain or weakness without increase in creatinine kinase. Myositis refers to muscle symptoms with increase in creatine kinase whereas in rhabdomyolysis, there are severe muscle symptoms with joint pain, marked weakness and ten fold rise in creatine kinase levels, usually with brown urine and urinary myoglobin [1-3]. In randomized,controlled trials, the incidence of nonspecific muscle pain or joint pain is similar (5\% each) in the intervention and control groups [13-17, 14-21]. However, some of the patients may have mild to moderate elevations of creatine kinase without muscle complaint. The temporal association with statin therapy of such adverse effects is enough to implicate that statin therapy may be the cause of these effects. Failure to discontinue statin therapy in these asymptomatic patients may cause further damage leading to rhabdomyolysis and acute renal necrosis. Statins may also cause an autoimmune myopathy requiring immunosuppressive treatment. The mechanisms of statin myotoxicity are unclear. If unrecognized in its early manifestations, complications from continued statin therapy may lead to rhabdomyolysis, hepatotoxicity, renal damage and death. Risk factors for myotoxicity include concomitant medication use and .medical conditions, and the patient's underlying genetic constitution. We review these considerations along with the recommended evaluation and treatment for patients presenting with statin myotoxicity.

\section{AMYOTROPHIC LATERAL SCLEROSIS}

While cases of amyotrophic lateral sclerosis (ALS) or ALS-like conditions have arisen in apparent association with HMG-CoA reductase inhibitors ('statins') and/or other lipidlowering drugs (collectively termed 'statins' in this paper for brevity), additional information is needed to understand whether the connection may be causal $[8,9]$. The University of California, San Diego (UCSD) Statin Effects Study is a patient-targeted adverse event surveillance project focused on lipid-lowering agents, whose aim is to capitalize on patient reporting to further define characteristics and natural history of statin adverse effects, and to ascertain whether a patient-targeted surveillance system might lead to presumptive identification of previously unrecognized adverse effects. ALS was a candidate 'new' adverse effect identified through this process. The aim of the analysis presented here was to examine characteristics and natural history of reported statin-associated ALS-like conditions with attention to factors that may bear on the issue of causality. For the present analysis, we focused on cases of statin-associated ALS that were reported to our study group prior to publication of a possible statin-ALS association. Of 35 identified subjects who had contacted the UCSD Statin Effects Study group to report ALS or an ALS-like condition, 18 could not be reached (e.g. contact information was no longer valid). Six were unable to participate (e.g. due to progression of their disease). Of the 11 who could be contacted and were able to participate, one declined to give informed consent. The remaining ten, with either a formal or probable diagnosis of ALS in the context of progressive muscle wasting/weakness arising in association with lipid-lowering drug therapy, completed a mail or phone survey eliciting information about ALS symptom onset and change in association with drug use/modification and development of statinassociated adverse effects. We reviewed findings in the context of literature on statin antioxidant/pro-oxidant balance, as well as ALS mechanisms involving oxidative stress and mitochondrial dysfunction. All ten subjects reported amelioration of symptoms with drug discontinuation and/or onset or exacerbation of symptoms with drug change, rechallenge or dose increase. Three subjects initiated coenzyme Q10 supplementation; all reported initial benefit. All subjects reportedly developed statin adverse effects (not indicative of ALS) prior to ALS symptom onset, strongly disproportionate to expectation ( $p<0.001)$. Since this reflects induction of prooxidant effects from statins, these findings lend weight to a literature-supported mechanism by which induction by statins of oxidative stress with amplification of mitochondrial dysfunction, arising in a vulnerable subgroup, may propel mechanisms underlying both adverse effects and, more rarely, ALS. A theoretical foundation and preliminary clinical observations suggest that statins (and other lipidlowering drugs) may rarely be associated with ALS in vulnerable individuals in whom pro-oxidant effects of statins predominate. Our observations have explanatory relevance extending to ALS causes that are not statin associated and to 


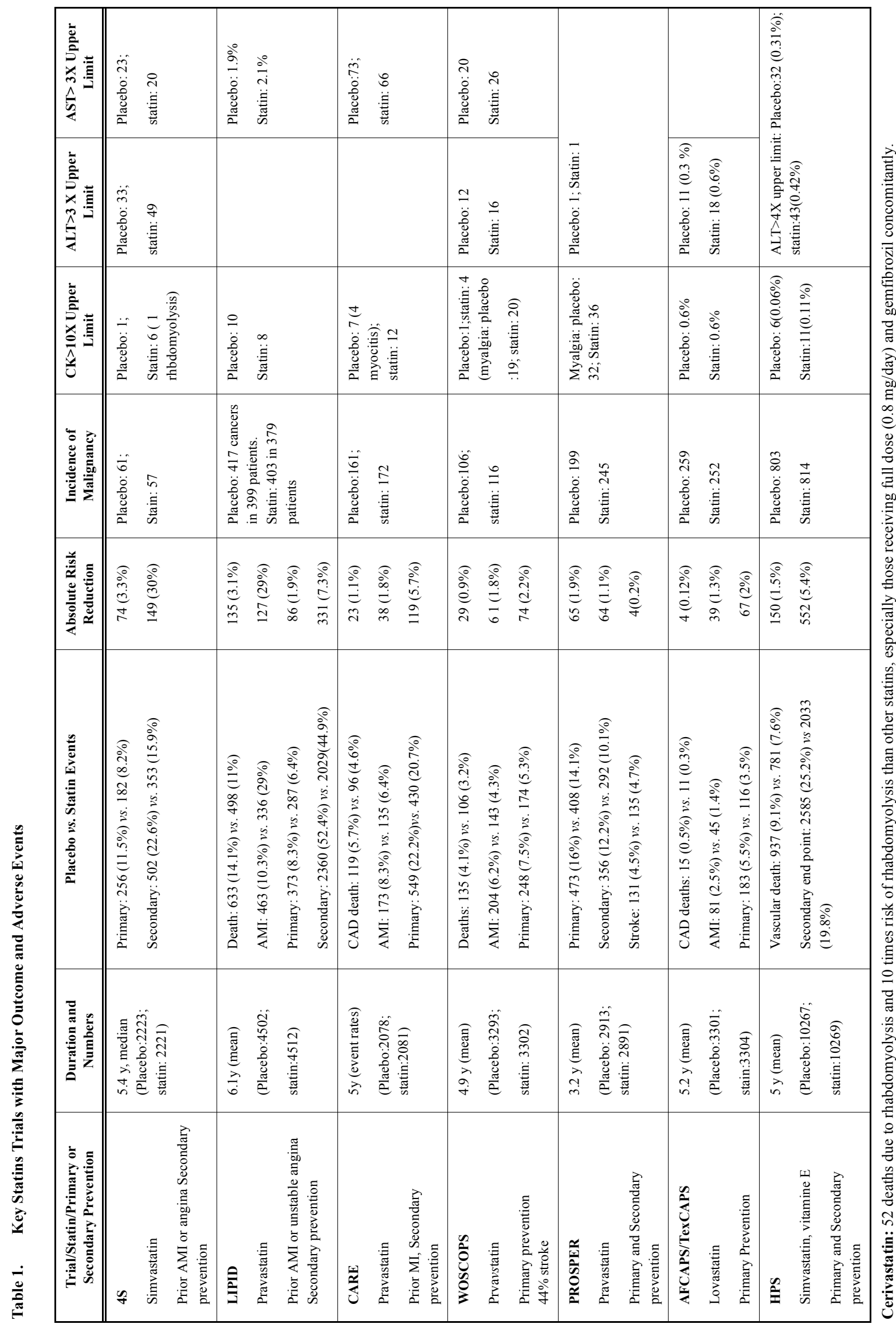




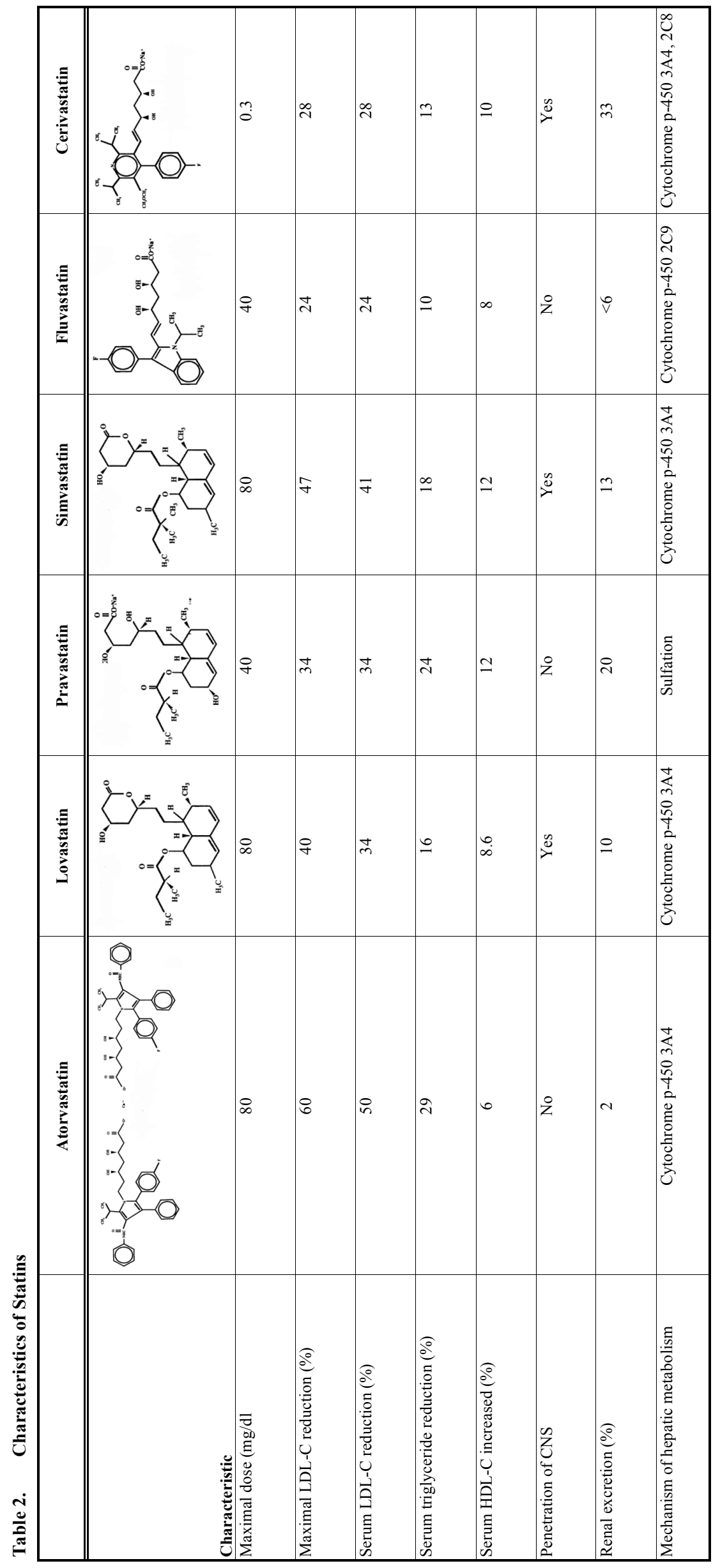


statin-associated neurodegenerative conditions that are not ALS. They suggest means for identification of a possible vulnerable subgroup. Indeed whether statins may, in contrast, confer ALS protection when antioxidant effects predominate merits examination.

A disproportionate reporting of amyotrophic lateral sclerosis (ALS) with HMG-CoA-reductase inhibitors (statins) in the Food and Drug Administration's (FDA) spontaneous adverse event reporting system has been observed [9]. The purpose was to describe the original ALS signal and to provide additional context for interpreting the signal by conducting retrospective analyses of data from long-term, placebo-controlled clinical trials of statins. The ALS signal was detected using the multi-item gamma Poisson shrinker (MGPS) algorithm. All cases of ALS reported in association with use of a statin were individually reviewed by two FDA neurologists. Manufacturers of lovastatin, pravastatin, simvastatin, fluvastatin, atorvastatin, cerivastatin, and rosuvastatin were requested to provide the number of cases of ALS diagnosed during all of their placebo-controlled statin trials that were at least 6 months in duration. There were 91 US and foreign reports of ALS with statins in adverse effect reporting system. The data mining signal scores for ALS and statins ranged from 8.5 to 1.6. Data were obtained from 41 statin clinical trials ranging in duration from 6 months to 5 years and representing approximately 200,000 patient-years of exposure to statin and approximately 200,000 patientyears of exposure to placebo. Nine cases of ALS were reported in statin-treated patients and 10 cases in placebotreated patients. Although we observed a data mining signal for ALS with statins in FDA's system, retrospective analyses of 41 statin clinical trials did not reveal an increased incidence of ALS in subjects treated with a statin compared with placebo.

\section{PULMONARY MANIFESTATIONS}

To systematically review all published case reports and the US Food and Drug Administration adverse event reporting (FDA-AER) database to examine the relationship between statins and interstitial lung disease [10] (ILD). PubMed (1987 to September 2007) and the FDA-AER database (as of June 2007) were searched for reports of ILD in which a statin was listed as a causative suspect. Two authors (one author for Pub Med cases and one for FDAAER cases) independently abstracted patient data. Given the paucity of information, all case reports and case series in English and French were included. All adverse event reports from the FDA-AER database in which a statin was listed as causative suspect were included. The literature search using PubMed yielded eight articles describing a total of 14 case reports of ILD in association with statin use. The FDA-AER system database contained 162 cases of reported statin-induced ILD as of June 2007. For every 10,000 reports of a statin-associated adverse event, approximately 1 to 40 reports were for ILD. Statin-induced ILD is a possible newly recognized side effect of statin therapy [10]. The mechanism of lung injury is not defined. The current review provides novel information from the FDA-AER that supports a possible, although unusual, pulmonary class effect of statins.

\section{PSYCHIATRIC MANIFESTATIONS}

The inhibitors of HMG-CoA reductase ('statins') are widely prescribed hypolipidaemic drugs, which have been evaluated in several clinical trials involving hundreds of thousands of patients [11]. However, recent interest has been focused on a potential risk of psychiatric ADRs associated with statins, including memory loss, depression, suicidality, aggression and antisocial behaviour. Special attention is currently being paid to the potential for statin-induced sleep disorders. To investigate the hypothesis that statins may be associated with psychiatric adverse events using quantitative and qualitative signal analysis. The Interregional Group of Pharmacovigilance database holds reports of suspected ADRs submitted since 1988 from eight Italian regions. In the present analysis, only reports ranked at least 'possible', according to WHO causality assessment criteria, were considered. Association between statins and psychiatric events was assessed by the case/non-case methodology, calculating the ADR reporting odds ratio (ROR) as a measure of disproportionality. Cases were defined as patients with at least one reported ADR combined with the system organ class (SOC) 'psychiatric disorders'. The non-cases comprised all patients who did not experience an ADR related to the SOC 'psychiatric disorders'. Index reports comprised all ADR reports involving at least one statin, while all ADR reports not involving statins as suspected drugs were used as controls. According to selection criteria, 35,314 reports were included in the analysis. A total of 71 psychiatric preferred terms combined with statins were identified in 60 reports. Among them, 14 reports $(23.3 \%)$ noted a positive rechallenge. Both the unadjusted $(0.8 ; 95 \%$ CI $0.6,1.1)$ and adjusted ROR $(0.7$; $95 \%$ CI $0.6,1.0)$ suggested a lower rate of reports of psychiatric events for statins as a whole class compared with all other drugs, although the difference was not significant. The five most frequently reported psychiatric events combined with statins were insomnia, somnolence, agitation, confusion and hallucination. Only insomnia was reported with higher frequency for statins compared with all other drugs $(\mathrm{ROR}=3.3 ; 95 \%$ CI $1.9,5.7)$, while confusion was reported with a lower frequency $(\mathrm{ROR}=0.4 ; 95 \%$ CI $0.1,0.9)$. Amongst statins available in Italy, only simvastatin (ROR = $0.5 ; 95 \%$ CI $0.2,0.9)$ showed a significantly lower rate of reports of psychiatric events compared with all other drugs together. A relatively small number of possible statinassociated psychiatric ADRs have been found in our database. No significant risks for a higher overall reporting of psychiatric ADRs associated with statins were identified in comparison with all other drugs combined. However, statinassociated insomnia resulted in a significant ROR that requires further investigation.

\section{OPHTHALMOLOGICAL MANIFESTATIONS}

To report the association between 3-hydroxy-3-methylglutaryl-CoA (HMG-CoA) reductase inhibitors (statins) and diplopia, blepharoptosis (ptosis), and ophthalmoplegia [12]. In this database study 256 subjects were studied. Spontaneous reports from the National Registry of Drug-Induced Ocular Side Effects, the World Health Organization (WHO), and the Food and Drug Administration were collected on statins and ptosis, diplopia, and ophthalmoplegia. Data garnered from the spontaneous reports include the type of statin, 
age, gender, adverse drug reaction (ADR), dosage, duration of therapy until onset of ADR, concomitant drugs, other systemic disease, and dechallenge and rechallenge data. Two hundred fifty-six case reports of ptosis, diplopia, and ophthalmoplegia associated with statins were reported, including 143 males, 91 females, and 22 case reports where the gender was not specified. The average age was $64.5+/-10$ years. Dosage varied between the different statin drugs, with the average dosage within the range recommended in the package insert for each different statin. Average time from beginning of therapy to appearance of the ADR was 8.3+/-1.5 months (range, 1 day- 84 months). Seven patients were taking 2 statin drugs and 5 also were taking gemfibrozil. Nine patients had diabetes mellitus. A total of 23 case reports described total ophthalmoplegia. Ptosis was reported alone 8 times and in conjunction with diplopia 18 times. There were 62 positive dechallenge and 14 positive rechallenge case reports. According to WHO criteria, the relationship between statin therapy and diplopia, ptosis, or ophthalmoplegia is possible. This causality assessment is based on the time relationship of drug administration and ADR development, the multiple positive dechallenge and rechallenge reports, and the plausible mechanism by which diplopia, ptosis, or ophthalmoplegia may occur: myositis of the extraocular muscles, the levator palpebrae superioris muscles, or both.

\section{ETIOLOGY AND MECHANISMS}

The etiology of statin myopathy remains unclear and concern about this toxicity is a leading reason that statins are underutilized. A number of observations suggest that this toxicity may be due to the metabolic effects of lipidlowering in patients with minor muscle disorders [44-46]. These patients have a high frequency of mutations for metabolic muscle diseases and often have depleted mitochondrial enzymes. Their exercise physiology and biopsy findings indicate reduced oxidation of fats and mitochondrial dysfunction. These subjects are often intolerant of other lipidlowering therapies in addition to statins, which suggests that the myopathy is due to lipid-lowering itself more than a simple pharmacokinetic reaction to high statin levels. Altogether, these findings support the concept that statin myopathy is a metabolic muscle disease.

HMG-CoA reductase inhibitors (statins) are a widely used class of drug, and like all medications, have potential for adverse effects. Here we review the statin adverse effects literature, first focusing on muscle effects as the most reported problem both in the literature and by patients. Evidence regarding the statin muscle effect mechanism, dose effect, drug interactions, and genetic predisposition is examined. We hypothesize, and provide evidence, that the demonstrated mitochondrial mechanisms for muscle effects have implications to other nonmuscle effects in patients treated with statins. In meta-analyses of randomized controlled trials (RCTs), muscle effects are more frequent with statins than with placebo. A number of manifestations of muscle effects have been reported, with rhabdomyolysis the most feared. Adverse effects are dose dependent, and risk is amplified by drug interactions that functionally increase statin potency, often through inhibition of the cytochrome P450 3A4 system $[40,41]$. An array of additional risk factors for statin toxicity are those that amplify (or reflect) mitochondrial or metabolic vulnerability, such as metabolic syndrome factors, thyroid disease, and genetic mutations linked to mitochondrial dysfunction. Converging evidence supports a mitochondrial foundation for muscle effects associated with statins, and both theoretical and empirical considerations suggest that mitochondrial dysfunction may also underlie many nonmuscle adverse manifestations of statins.

Evidence from RCTs and studies of other designs indicates existence of additional statin-associated effects, such as cognitive loss, neuropathy, pancreatic and hepatic dysfunction, and sexual dysfunction. Physician awareness of statin effetcs is reportedly low even for the adverse effects, most widely reported by patients. The mechanism of statin toxicity may be cell damage due to oxidation and decrease in antioxidant protection.

\section{PREVENTION AND MANAGEMENT}

Approximately $10 \%$ of patients treated with statins experience some form of muscle-related side effects in clinical practice. These can range from asymptomatic creatine kinase (CK) elevation, to muscle pain, weakness, and its most severe form, rhabdomyolysis. The cause of statin myopathy is presumed to be the same for its variable presentation but has not been defined. In patients with myopathic symptoms, their symptoms and CK levels determine whether statin therapy can be continued or must be stopped.

Intensive lipid modification using statins(3-hydroxy-3methylglutaryl coenzyme A(HMG-CoA) reductase inhibitors)has been associated with a substantial reduction of cardiovascular morbidity and mortality. Interestingly, this decade of 1990s has been christined "the statin decennial" and statins are proclaimed the wonder drugs, influencing a wide range of physiological, biochemical and biological functions. This list appears to be quite large and includes several pleitropic benefits apart from its lipid modification effects. These actions are vasodilative, antithrombotic, antioxidant, antiinflammatory, antiproliferative, anticoadulant, angiogenic and bone formation inducing functions of statins. Unfortunately, several of these beneficial effects have not been substantiated by randomized, controlled trials and only a few investigators consider some of these beneficial effects as controversial [3]. Awareness and vigilance for these adverse manifestations should be maintained to enable informed treatment decisions, treatment modification if appropriate, improved quality of patient care, and reduced patient morbidity.

\section{POSSIBLE ROLE OF ANTIOXIDANTS}

Statin therapy may also decrease antioxidant status in the body, resulting in to increase in free radical damage to cells in various parts of body; liver, nerves, muscles. Statins can decrease natural antioxidant protection present in our body and predispose toxicity [4-12]. The antioxidant status of our body depends on the presence of coenzyme Q10, vitamin A, $\mathrm{E}$, and $\mathrm{C}$ and beta- carotine, superoxide dismutase, catalase and ceruloplasmin. Apart from these antioxidants, flavonoids, anthrocyclins, minerals; selinium, chromium and copper and w-3 fatty acids may also provide protection against oxidative stress predisposed by statins [18-20]. In one study $[8,9]$, three subjects initiated coenzyme Q10 supplementation; all reported initial benefit. All subjects reportedly developed statin adverse effects (not indicative of ALS) 
prior to ALS symptom onset, strongly disproportionate to expectation $(\mathrm{p}<0.001)$. Since this reflects induction of pro-oxidant effects from statins, these findings lend weight to a literature-supported mechanism by which induction by statins of oxidative stress with amplification of mitochondrial dysfunction, arising in a vulnerable subgroup, may propel mechanisms underlying both adverse effects and, more rarely, ALS. Several small scale reports indicate that statins can cause myopathies and rhabdomyolysis with renal failure, and increase in transeminases due to cell damage [310]. It is suggested that prior or simultaneous administration of antioxidants may be protective against P450 induced cell damage by the statins, resulting in to decrease in statin toxicity.

Coenzyme Q10 is omnipresent substance serving also like coenzyme in mitochondrial phosphorylation. It should be synthetised by endogenous way from mevalonateisoprene like a result of HMG-CoA reductase activity [26]. Thus,it is not surprising that coenzyme Q10 concentrations are decreased during statin therapy due to both decrease of LDL cholesterol and inhibition of its synthesis. It was shown that during statin therapy significant decrease of coenzyme Q10 was observed (up to $40 \%$ ) and subsequent disorder of mitochondrial energetic metabolism may lead to myopathy [41]. The reduction in serum coenzyme Q10 may be dose related and so may be the muscle damage [42]. To establish the extent to which atorvastatin inhibits Coenzyme Q10 synthesis, 34 hypercholesterolemic patients were administered $80 \mathrm{mg} /$ day of atorvastatin for 14 or 30days [43]. Mean coenzyme Q concentration decreased by $49 \%$ after 14days of atorvastatin therapy and $52 \%$ after 30 of treatment. Baseline coenzyme Q level was $1.26 \mathrm{ug} / \mathrm{ml}$ and after 30days it was $0.62 \mathrm{ug} / \mathrm{ml}$.

In London, the global television network $\mathrm{CNN}$ announced in a dispatch on January 18, 2002, that the deaths resulting from treatment of hypercholesterolemia with cerivastatin (Baycol)now have exceeded a century. Subsequently, the Wall Street Journal, January 21,2002) reported that Bayer AG has given similar indications about deaths linked to Baycol. The purpose is to provide warning about inappropriate use of statins and updated recommendations for its proper use including cautions, contraindications and safety monitoring for therapy with this group of agents. The International College of Cardiology discussed this issue of cerivastatin intoxication in their meeting in April 2002 and once more, the present situation, in November 2004 in its executive meeting and press release, at Taipei,Taiwan during the 3rd International Congress on Cardiovascular Disease, as to what are the causes of this adverse manifestations and how to decrease the adverse effects of this great terminator of atherosclerosis in developed and developing economies. The role of coenzyme Q10 among patients receiving statin was reemphasised in the 5 th ICCD at Kosice, Slovakia on June 4-7, 2009.

\section{CONTROL OF OTHER RISK FACTORS FOR PREVENTION OF MYOPATHY}

It is important to treat blood pressure and use other methods for treatment of dyslipidemia to avoid excessive use of statins [44-48]. More than one billion people in the world may have serum cholesterol levels where statins may be indicated. Therefore, any intoxication of statins, despite its low occurrence may become a public concern. According to one estimate, worldwide sales of hypocholesterolemic and hypotriglyceridemic agents reached $\$ 15.9$ million in 2001 which might increase to 36 million in United States alone with publication of new guidelines for Americans [28]. Approximately, 5 of the 14 expert members declared financial relations with upto 11 pharmaceutical companies which may indicate the use of individual influence on the guidelines, to some of us [3]. However, almost all of these investigators are the best in the field and are known for their integrity and academic honesty. Moreover, this report [28], has been revised [1-3] which includes recent information on myopathy compiled by the FDA as well as information from the clinical trials. There is uniform agreement that reduction of total and low density lipoprotein(LDL) cholesterol reduces cardiovascular risk which is reflected in the aggressive approach advocated in the current guidelines [28]. It is possible that the current emphasis on lipid management would continue in the United States as well as it is likely to be followed in other developed and developing countries [31].

American College of Cardiology/American Heart Association/ National Heart Lung and Blood Institute (ACC/AHA/NHLBI)have discussed in May 2002 and have published an Advisory [31] with the intention to summarize professionals, the current understanding of statin use, focussed on myopathy. The NCEP guidelines are endorsed by the American Heart Association as earlier, as well as American College of Cardiology this time [46]. This report is based on rigourous clinical trial evidence to identify additional high risk subjects for treatment, causing marked increase in the number of patients who may be candidates for statin administration. Apart from CAD, other forms of atherosclerotic disease, diabetes mellitus, presence of multiple risk factors and severe hypercholesterolemia are important indications for statin therapy according to new guidelines. In several of these situations one noteworthy advantage of these guidelines is that many patients with borderline increase in cholesterol would be treated with smallest dose of statin whereas in some patients, relatively high doses of statins or a combination therapy will be required to achieve the goals of treatment. The International College of Cardiology emphasizes on catching patients at early stage of LDL increase $(>90 \mathrm{mg} / \mathrm{dl})$ in presence of other risk factors such as hypertension or type 2 diabetes or inflammation (hsCRP>2.0mg/ dl) to administer lowest dose of statin, rather than waiting to treat severe hypercholesterolemia with higher doses and combinations [29]. There are some parallels with the treatment of arterial hypertension. In contrast with JNC I, different classes of drugs are now recommended by JNC VI like drugs of the first choice even administered in combination preferentially in low dosage [44-46]. During last few years, there is strong tendency in clinical trials towards use of higher doses of statins with the aim of achieving target levels of lipid profile. These target levels should be achieved in many patients also with lower doses of statins used in monotherapy or in combination but with different groups of drugs including ezetimibe [45] or antithrombotic substatnces like sulodexide which has also beneficial effects on lipid profile [47]. 


\section{MEDITERRANEAN DIET}

There is still need to emphasize the role of Mediterranean diet or Indo-Mediterranean diet rich in w-3 fatty acids, which can cause a decrease of atherogenic lipids as well as decrease of cardiovascular morbidity and mortality [48]. Lipid lowering diet offer significant chance to decrease dose of statins used in monotherapy or in combination. To prevent side effects of statins including myopathy, it should be useful to combine statins with coenzyme Q10, however, greater clinical studies are needed. The secondary targets of therapy such as hypertriglyceridemia, low high density lipoprotein(HDL) cholesterol and high LDL+very low LDL and inflammation are also identified for treatment by the NCEP panel causing a further increase in the use of lipid lowering agents. The role of lipids and inflammation, in vascular disease in diabetes has been established, which would further enhance the use of statins and combination among patients with diabetic dyslipidemia $[49,50]$. In a randomized,controlled intervention trial, among 71patients (Intervention group) and 73 patients(Control group) of acute myocardial infarction(AMI), who were administered coenzyme Q10 $(120 \mathrm{mg} /$ day) or B vitamins for one year, half of the patients(n=36 vs 31$)$ in both groups, received lovastatin(10-20mg/day) [51]. Adverse effects, such as nausea(30.1 vs $9.8 \%)$, vomiting(13.7 vs $11.2 \%$ ), were more common in the coenzyme $\mathrm{Q}$ group whereas fatigue ( 6.8 vs $40.8 \%, \mathrm{P}<0.01$ ) was more common in the control group [51]. Fatigue is an early manifestation of muscle damage, indicating that coenzyme $\mathrm{Q}$ may have prevented this adverse effect of lovastatin in the intervention group. Therefore, it is necessary to pay greater attention on efficacy, safety and cost-effectiveness of lipid lowering regimen including statins and the role of coenzyme Q10 and the diet and physical activity in the prevention of statin intolerance.

\section{WHICH ONE OF THE STATINS IS SUPERSTATIN}

All of the statins are powerful LDL lowering agents that are widely used in all the countries to improve cardiovascular risk profiles. Randomized, controlled, clinical trials have demonstrated a decline in CAD and total mortality, reductions in myocardial infarctions, revascularization procedures, stroke and peripheral vascular disease during a mean duration of treatment for approximately 5.4 years [30-37]. The safety of treatment with all of the statins, outside of clinical trials has not been fully studied. It is likely that the incidence of adverse effects, may be higher in clinical situations where patients are less carefully monitored as compared to side effects watch during clinical trials.

Fatal rhabdomyolysis is 16 to 80 fold greater for cerivastatin compared to other statin [22]. Since Bayer AG indicated that 100 deaths appear to be linked to Baycol, therefore, it has withdrawn cerivastatin (Baycol) from the market. At the time of withdrawal, the Food and Drug Administration of the United States had received reports of 31 deaths in US alone due to rhabdomyolysis associated with the use of cerivastatin, 12 of which involved concomitant administration of gemfibrozil. Despite all positive effects of statins as demonstrated in large clinical trials, there is still gap between use of statins in clinical practice and statin evidence based medicine. There are some possible explanations: Firstly, there is always some delay after publishing of the results of the trials. Secondly, in clinical trials there are evaluated selected subgroups of patients, safety cautions are more strict, that is why many doctors are afraid of possible side effects of lipid lowering therapy in their common clinical practice. Thirdly, following evidence based medicine, cardiovascular diseases and mainly coronary artery disease imposes a massive economic burden not only for developing countries but also for highly developed countries, e.g. in the United States there were in 1998 about $28 \%$ of adults eligible for lipid regulating therapy according to earlier NCEP II guidelines [46]. Lipid lowering therapy is considered life long therapy and from this point of view we are still missing reliable informations, especially long-term safety data for statins.

\section{ROSUASTATIN: THE SUPERSTATIN}

Rosuastatin is a safe and effective treatment for elevated cholesterol. A thorough analysis of the post-marketing safety data involving an estimated 1.6 million patients and 4.4 million prescriptions show CRESTOR to have a similar safety profile to that of other marketed statins. This is in line with the clinical trial database and current US CRESTOR labeling. Rosuvastatin represents an advance on the pharmacologic and clinical properties of other agents in this class $[13,14,52-56]$. Compared to other statins, it possesses a greater number of binding interactions with HMG-CoA reductase and has a high affinity for the active site of the enzyme. Rosuvastatin is relatively hydrophilic and is selectively taken up by, and active in hepatic cells. It has the longest terminal half life of the statins and is only minimally metabolized by the cytochrome P450 (CYP 450) enzyme system with no significant involvement of the 3A4 enzyme. There is absence of clinically significant drug interactions, between rosuvastatin and other drugs known to inhibit CYP 450 enzymes. The molecule is superior to atorvastatin, simvastatin, and pravastatin in reducing LDL-C and favourably modifying other components of the atherogenic lipid profile such as inflammation. In patients with hypercholesterolemia and dyslipidemia and the metabolic syndrome, treatment with rosuvastatin should help to improve lipid goals and reduce the requirement for dose titration. In a Randomized, double blind crossover trial of rosuvastatin $(40 \mathrm{mg} /$ day for 8 weeks)on atherogenic apolipoprotein B-containing lipoprotein subfractions, the effects were studied at Glasgow Royal infirmary [53]. Rosuvastatin was found to reduce cholesteryl easter transfer protein by $33 \%$ in normotrigly- ceridemic and $37 \%$ in hypertriglyceridemic subjects (both $\mathrm{P}<0.001$ )whereas a reduction in cholesteryl ester transfer activity was observed in later only.

The JUPITER study screened almost 90, 000 healthy volunteers aged 50 years or more for participation in this trial among healthy subjects $[13,14]$. One in five subjects [17] of those screened, were included in the trial. Treatment with statin is not indicated in such individuals because they had no history of CAD, and their LDL-cholesterol levels were below the usual threshold for treatment of $130 \mathrm{mg} / \mathrm{dl}$ $(3.33 \mathrm{mmol} / \mathrm{L})$. The hypothesis was that hsCRP is helpful in identifying individuals at high risk, and only those participants with hsCRP levels of $2.0 \mathrm{mg} / \mathrm{dl}$ or more were selected for this study. Furthermore, given that statins reduce high levels of hsCRP, and also possibly other cytokines, the investigators expected to see a bonus of reduced inflammation through CRP reduction. This trial was prematurely discon- 
tinued, after 1.9 years, because rosuvastatin $20 \mathrm{mg}$ daily had reduced LDL-cholesterol levels by about 50\% in comparison with placebo. Despite the fact that levels of LDL cholesterol and hsCRP were only slightly correlated in individual patients, those who achieved a dual treatment target of LDL cholesterol lower than $1.8 \mathrm{mmol} / \mathrm{l}(70 \mathrm{mg} / \mathrm{dl})$ and hsCRP lower than $2 \mathrm{mg} / \mathrm{l}$ had a $65 \%$ reduction in CVD events, compared with a $33 \%$ reduction in patients who achieved one or neither target. The authors reported that participants who achieved an LDL cholesterol level of below $1.8 \mathrm{mmol} / \mathrm{l}$ (70 $\mathrm{mg} / \mathrm{dl}$ ) had a 55\% reduction in CVD events and those who achieved a hsCRP reduction of below $2 \mathrm{mg} / \mathrm{l}$, a $62 \%$ reduction, which is slightly greater benefit compared to LDL reduction. However, patients who achieved the more stringent target of a LDL cholesterol level of below $1.8 \mathrm{~m} \mathrm{~mol} / 1$ (70 $\mathrm{mg} / \mathrm{dl}$ ) and a hsCRP reduction of below $1 \mathrm{mg} / \mathrm{l}$ had a $79 \%$ reduction in CVD events. This shows that decrease in inflammation in conjunction with LDL reduction has greater benefit at the same level of LDL reduction, which appears to be due to a further decrease in oxidized LDL. There is a need to measure oxidized LDL as well as total LDL to find out the mechanism of this interaction. Although, these substantial reductions in LDL and hsCRP were not translated into significant reduction in cardiovascular deaths in the intervention group compared to placebo group (12 vs 12 deaths), the benefits highlighted in JUPITER have been considered great. The case fatality rate due to MI was greater in the rosuastatin group compared to placebo group ( $29.0 \%$ vs $8.8 \%), \mathrm{n}=9$ vs 6) after 1.9 years of treatment.

International College of Cardiology [45] would strongly support recommendations of NCEP ATP III to treat elevated atherogenic lipids to their target levels and to guarantee safety of such therapy (5th ICCD, Kosice, Slovakia Nov 2009). Possible risks of widely prescribed statin therapy should be decreased by administering of lower doses, combination with coenzyme Q10 and avoidance of risky combinations of drugs with interfering metabolism via cytochrome $\mathrm{P}$ 450. The role of lipid lowering diet plus exercise is indisputable and may support lower doses of lipid lowering drugs or even lower use of these drugs [48] and may prevent prooxidative stage that is important for statin intolerance. Use of coenzyme Q10 even in smaller doses of $30 \mathrm{mg} /$ day may be rewarding in the prevention of adverse effects of statins [1, 51]. Claims of Bayer are logical, that physicians using cerivastatin are equally responsible for the statin intoxication [41]. Physicians should have studied the literature given by Bayer carefully before prescribing and should have instructed the patients to come for advise more frequently especially when higher doses or combinations are being prescribed. It is not clear,how far patients can be responsible for the toxicity when they do not follow the doctors advise on scheduled visit.

\section{AVOIDING COMBINATIONS}

Statin therapy effectively prevents vascular disease, but treatment targets are often not achieved. In one recent metaanalysis [57], the benefits and harms of high-dose statin monotherapy with those of combination therapy in adults at high risk for coronary disease were compared. Data sources were english-language records from MEDLINE (19662009), EMBASE (1980-2009), and the Cochrane Library (third quarter of 2008). A reviewer screened records, and a second reviewer verified selection of randomized, controlled trials in adult patients that compared combinations of statins and bile-acid sequestrants, fibrates, ezetimibe, niacin, or omega-3 fatty acids with statin monotherapy, as well as nonrandomized comparative studies that were longer than 24 weeks and reported clinical and harms outcomes. Data were abstracted for studies by using standardized forms, and study quality was rated with a standardized scale and strength of evidence by using the grading of recommendations assessment, development, and evaluation approach which met 102 studies by eligibility criteria. The main analysis compared combination therapy with high-dose statin monotherapy in high-risk patients. Very-low-strength evidence showed that statin-ezetimibe ( 2 trials; $\mathrm{n}=439$ ) and statin-fibrate ( 1 trial; $\mathrm{n}=166)$ combinations did not reduce mortality more than high-dose statin monotherapy. No trials compared the effect of combination therapy versus high-dose statin monotherapy on the incidence of myocardial infarction, stroke, or revascularization procedures. Two statin-ezetimibe trials $(n=295)$ demonstrated higher low-density lipoprotein cholesterol goal attainment with combination therapy (odds ratio, 7.21 [95\% CI, 4.30 to 12.08$]$ ). Trials in lower-risk patients did not show a difference in mortality. Limitations: Studies were generally short, focused on surrogate outcomes, and were heterogeneous in study sample risk for coronary disease. Only a few studies examined treatment combinations other than statinezetimibe. Limited evidence suggests that combinations of lipid-lowering agents do not improve clinical outcomes more than high-dose statin monotherapy. Very-low-quality evidence favors statin-ezetimibe treatment for attainment of low-density lipoprotein cholesterol goals. It ispossible that avoiding combinations with other agents can help in the prevention of statin toxicity.

In brief, statins appear to be safe drugs, if used in smaller doses along with diet and lifestyle modification. There is a need to consider coenzyme Q10 and w-3 fatty acd administration in conjunction with statin when they are given > $20 \mathrm{mg} / \mathrm{day}$, as it might also prevent deaths due to myopathy and other toxic effects because statin toxicity is considered a metabolic myopathy [41,58]. It is better to spend than to die, by avoiding the use of coenzyme Q10, especially those taking combinations which are now commonly used widely due to presence of metabolic syndrome and metabolic myopathy [54-58]. Use of other antioxidants and Mediterranean diet or designer foods need further studies, to demonstrate their role in the prevention of statin intolerance.

\section{ACKNOWLEDGEMENTS}

We are grateful to Mr Raj Chopra, President Tishcon Corporation,Westbury, NY, USA for support for this study. We are also grateful to International College of Nutrition and International College of Cardiology for their input.

\section{REFERENCES}

[1] Chiang CA, Pella D, Singh RB. Adverse effects of hmg coareductase inhibitors and the role of coenzyme q10. J Nutr Environ Med 2004; 14: 17-28.

[2] Bełtowski J, Wójcicka G, Jamroz-Wiśniewska A. Adverse effects of statins - mechanisms and consequences. Curr Drug Saf 2009 Sep 1. (Epub ahead of print)

[3] Joy TR, Hegele RA. Narrative review: statin-related myopathy. Ann Intern Med 2009; 150(12): 858-68. 
[4] Vladutiu GD. Genetic predisposition to statin myopathy. Curr Opin Rheumatol 2008; 20: 648-55.

[5] Radcliffe KA, Campbell WW. Statin myopathy. Curr Neurol Neurosci Rep 2008; 8: 66-72.

[6] Venero CV, Thompson PD. Managing statin myopathy. Endocrinol Metab Clin North Am 2009; 38: 121-36.

[7] Phillips PS, Haas RH. Statin myopathy as a metabolic muscle disease. Expert Rev Cardiovasc Ther 2008; 6: 971-8.

[8] Golomb BA, Kwon EK, Koperski S, Evans MA. Amyotrophic lateral sclerosis-like conditions in possible association with cholesterol-lowering drugs: An Analysis of PatientReports to the University of California, San Diego (UCSD) Statin Effects Study. Drug Saf 2009; 32: 649-61.

[9] Colman E, Szarfman A, Wyeth J, et al. An evaluation of a data mining signal for amyotrophic lateral sclerosis and statins detected in FDA's spontaneous adverse event reporting system. Pharmacoepidemiol Drug Saf 2008; 17: 1068-76.

[10] Fernández AB, Karas RH, Alsheikh-Ali AA, Thompson PD. Statins and interstitial lung disease: a systematic review of the literature and of food and drug administration adverse event reports. Chest 2008; 134: 824-30.

[11] Tuccori M, Lapi F, Testi A, et al. Statin-associated psychiatric adverse events: a case/non-case evaluation of an Italian database of spontaneous adverse drug reaction reporting. Drug Saf 2008; 31: 1115-23.

[12] Fraunfelder FW, Richards AB. Diplopia, blepharoptosis, and ophthalmoplegia and 3-hydroxy-3-methyl-glutaryl-CoA reductase inhibitor use. Ophthalmology 2008; 115: 2282-5.

[13] Ridker PM, Danieison E, Fonseka FAH, et al. Reduction in Creactive protein and LDL cholesterol and cardiovascular event rates after initiation of rosuvastatin: a prospective study of the JUPITER trial. Lancet 2009; 373: 1175-82.

[14] Ridker PM, Danieison E, Fonseka FAH, et al. Rosuvastatin to prevent vascular events in men and women with elevated Creactive protein. N Engl J Med 2008; 359: 2195-202.

[15] Nissen SE, Tuzcu EM, Shoenhagen P, et al. REVERSAL Investigators.Effect of intensive compared with moderate lipid lowering therapy on progression of coronary atherosclerosis. A randomized, controlled trial. JAMA 2004; 291: 1071-80.

[16] Cannon CP, Braunwald E, MeCabe CH, Redar DJ. Pravastatin or Atorvastatin evaluation and infection therapy. Thrombolysis in Myocardial Infarction 22 Investigators. Intensive versus moderate lipid lowering with statins after acute coronary syndromes. N Engl J Med 2004; 350: 1495-504.

[17] Baigent C, Keech A, Kearney PM, et al. Efficacy and safety of cholesterol-lowering treatment: prospective meta-analysis of data from 90,056 participants in 14 randomised trials of statins. Lancet 2005; 366: 1267-78

[18] Koumis T, Nathan JP, Rosenberg JM, Cicero LA. Strategies for the prevention and treatment of statin-induced myopathy:is there a role for ubiquinone supplementation? Am J Health Syst Pharmacol 2004; 61: 515-9.

[19] Chong P, Seeger J, Franklin C. Clinically relevant differences between the statins:implications for therapeutic selection. Am J Med 2001; 111: 390-400.

[20] Bliznakov EG. Lipid lowering drugs(statins),cholesterol,and coenzyme Q10-The Baycol case-a modern Pandora's box. Biomed Pharmacother 2002; 56: 56-9.

[21] Gaist D, Rodriguez LA, Huerta C, Hallas J, Sindrup SH. Lipid lowering drugs and risk of myopathy:a population based follow up study. Epidemiology 2001; 12: 565-69.

[22] Staffa JA, Green L. Cerivastatin and reports of rhabdo-myolysis. N Engl J Med 2002; 346: 565-40.

[23] Gaist D, Jeppesen U, Anderson M, García Rodríguez LA, Hallas J, Sindrup SH. Statins and risk of polyneuropathy: A case control study. Neurology 2002; 58: 1333-37.

[24] Farmer JA. Learning from the cerivastatin experience. Lancet 2001; 358: 1383-85.

[25] Pierce LR,Wysowski DK, Gross TP. Myopathy and rhabdomyolysis associated with lovastatin-gemphibrozil combination therapy. JAMA 1990; 264: 71-5.

[26] Pedersen TR, Tobert JA. Benefits and risks of HMG-CoA reductase inhibitors in the prevention of coronary heart disease: a reappraisal. Drug Saf 1996; 14: 11-24.

[27] Hsu I, Spinler SA, Johnson NE. Comparative evaluation of the safety and efficacy of HMG-CoA reductase inhibitors monotherapy in the treatment of primary hypercholesterolemia. Ann Pharmacother 1995; 29: 743-59.

[28] Cleeman JI. Executive summary of the third Report of the National Cholesterol Education Program(NCEP). Expert Panel on detection,evaluation, and treatment of high blood cholesterol in adults. JAMA 2001; 285: 2486-97.

[29] Pella D, Singh RB,Tomlinson B, Kong CW. Coronary artery disease in developing and newly industrialized countries: a scientific statement of the International College of Cardiology. In: Choklingham A, Berkowitz I, Singal PK, Dhalla NS, Eds. Frontiers in Cardiovascular Health. Boston, USA: Kluwer Academic Publishers 2003; pp. 473-88.

[30] Downs JR, Clearfield M, Weis S, et al. Primary prevention of acute coronary events with lovastatin in men and women with average cholesterol levels:results of AFCAPS/TexCAPS. Air Force/Texas Coronary Atherosclerosis Prevention Study. JAMA 1998; 279: 1615-22.

[31] Shepherd J, Cobbe SM, Ford I, et al. Prevention of coronary heart disease in men with hypercholesterolemia. West of Scotland Coronary Prevention Study Group. N Engl J Med 1995; 333: 13017.

[32] Sacks FM, Pfeffer MA, Moye LA, et al. The effect of pravastatin on coronary events after myocardial infarction in patients with average cholesterol levels.Cholesterol and Recurrent Events Trial Investigators. N Engl J Med 1996; 335: 1001-9.

[33] Wascher TC. Long-term statin safety and efficacy in secondary prevention: can combination therapy improve outcomes?Atheroscler Suppl 2003; 4(5): 11-6.

[34] Scandinavian Simstatin Survival Study Group. Randomized trial of cholesterol lowering in 4444 patients with coronary heart disease:the Scandinavian Simvastatin Survival Study(4S). Lancet 1994; 344: 1383-9.

[35] LIPID Study Group. Prevention of cardiovascular events and death with pravastatin in patients with coronary heart disease and a broad range of initial cholesterol levels. The Long-Term Intervention with Pravastatin in Ischemic Disease(LIPID) Study Group. N Engl J Med 1998; 339: 1349-57.

[36] Shepherd J and PROSPER Study Group. Pravastatin in elderly individuals at risk of vascular disease(PROSPER):a randomized, controlled trial. Lancet 2002; 360: 1623-30.

[37] Heart Protection Study Collaborative Group. MRC/BHF Heart Protection Study of individuals:cholesterol lowering with simvastatin in 20,536 high-risk a randomized,placebo controlled trial. Lancet 2002; 360: 7-22.

[38] Sever PS, Dahlof B, Poulter NR, et al. Prevention of coronary and stroke events with atorvastatin in hypertensive patients who have average or lower-than-average cholesterol concentrations, in the Anglo-Scandinavian Cardiac Outcomes Trial-Lipid Lowering Arm(ASCOT-LLA):a multicentre randomized controlled trial. Lancet 2003; 361: 1159-58.

[39] Nelson DR, Katamaki T, Waxman DL, et al. The P450 superfamily: update on new sequences, gene mapping, accession numbers, early trivial names of enzymes and nomenclature. DNA Cell Biol 1993; 12: 1-51.

[40] Nebert DW. The P450 superfamily: update on new sequences, gene mapping and recommended nomenclature. DNA Cell Biol 1991; 10: $1-14$

[41] London SF, Gross KF, Ringel SP. Cholesterol lowering agent myopathy. Neurology 1991; 41: 1159-60.

[42] Mortensen SA, Leth A, Agner E, Rohde M. Dose-related decrease of serum coenzyme Q10 during treatment with HMG CoA reductase inhibitors. Mol Aspects Med 1997; 18(Suppl S): S13744.

[43] Rundek T. Atovastatin inhibits coenzyme Q synthesis. Arch Neurol 2004; 61: 889-92.

[44] Joint National Committee on Detection, Evaluation, and Treatment of High Blood Pressure. The 1984 report of the Joint National Committee on Detection, Evaluation, and Treatment of High Blood Pressure. Arch Intern Med 1984; 144: 1045-57.

[45] Pella D, Rybár R, Valočik G, Petrášová D, Trejbal D, Mitro P. Secondary prevention of atherosclerosis by combined treatment with sulodexide and fluvastatin. Cardiovascular Diseases Bologna: Monduzzi Editore 2002; pp. 289-93.

[46] ACC/AHA/NHLBI. Clinical advisory on statins: Clinical advisory on the use and safety of statins. J Am Coll Cardiol 2002; 40: 56772 . 
[47] Chang JT, Staffa JA, Parks M, Green L. Rhabdomyolysis with HMG-CoA reductase inhibitors and gemphibrozil combination therapy. Phamacoepidemiol Drug Saf 2004; 13: 417-26.

[48] Singh RB, Dubnov O, Niaz MA, et al. The effect of an indomediterranean diet on the progression of coronary artery disease in high risk patients . The Indo-Mediterranean Diet Heart Study. Lancet 2002; 360: 1455-61.

[49] Haffner SM. Statin therapy for the treatment of diabetic dyslipidemia. Diabetes Metab Res Rev 2003; 19: 280-87.

[50] Sommerjer DW,Van Zanten AP, MacGillavry MR, Meijers JM, Reitsma PH, Cate HT. Antiinflammatory and anticoagulant effects pravastatin in patients with type 2 diabetes. Diabetes Care 2004; 27: 468-73.

[51] Singh RB, Neki NS, Kartikey K, et al. Effect of coenzyme Q10 on risk of atherosclerosis in patients with recent myocardial infarction. Mol Cell Biochem 2003; 246: 75-82.

[52] Rosenson RS. Rosuvastatin:a new inhibitor of HMG-CoA reductase for the treatment of dyslipidemia. Expert Rev Cardiovasc Ther 2003; 1: 495-505.
[53] Caslake MJ, Stewart G, Day SP, et al. Phenotype dependent and independent actions of rosuvastatin on atherogenic lipoprotein subfractions in hyperlipidemia. Atherosclerosis 2003; 171: 245-53.

[54] Walley T, Follino-Gallow P, Schwabe U, van Ganse E, EuroMedStat group. Variations and increase in use of statins across Europe: data from administrative databases. BMJ 2004; 328: 385-6.

[55] Wolfe AM. Dangers of rosuvastatin identified before and after FDA approval. Lancet 2004; 363: 2189-90.

[56] Coordinating Committee of the National Cholesterol Education Program.Implications of recent clinical trials for the National Cholesterol Education Program Adult Treatment Panel III Guidelines. Circulation 2004; 110: 227-39.

[57] Sharma M, Ansari MT, Abou-Setta AM, et al. Systematic review: Comparative effectiveness and harms of combinations of lipidmodifying agents and high-dose statin monotherapy. Ann Intern Med 2009; 151(9): 622-30.

[58] Golomb BA, Evans MA. Statin adverse effects: a review of the literature and evidence for a mitochondrial mechanism. Am J Cardiovasc Drugs 2008; 8: 373-418.

(C) Fedacko et al.; Licensee Bentham Open.

This is an open access article licensed under the terms of the Creative Commons Attribution Non-Commercial License (http://creativecommons.org/licenses/ by-nc/3.0/) which permits unrestricted, non-commercial use, distribution and reproduction in any medium, provided the work is properly cited. 